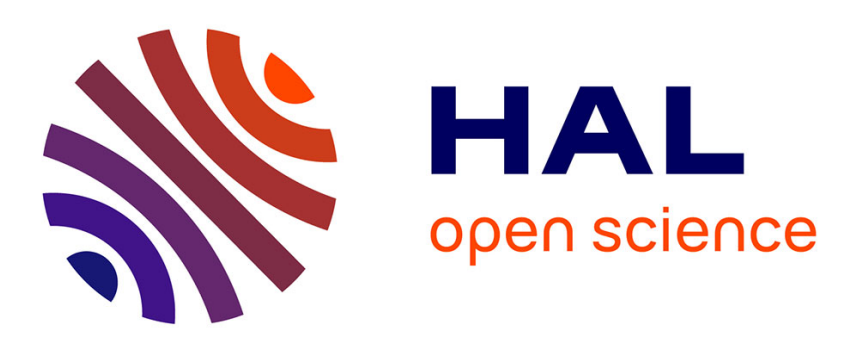

\title{
Continuous-Discrete Time Observer for a class of MIMO Nonlinear Systems
}

\author{
Mondher Farza, Mohammed M'Saad, Mamadou Lamine Fall, Eric Pigeon, \\ Olivier Gehan, R. Mosrati
}

\section{- To cite this version:}

Mondher Farza, Mohammed M'Saad, Mamadou Lamine Fall, Eric Pigeon, Olivier Gehan, et al.. Continuous-Discrete Time Observer for a class of MIMO Nonlinear Systems. IEEE Transactions on Automatic Control, 2014, pp.1060 - 1065. 10.1109/TAC.2013.2283754 . hal-01062739

\section{HAL Id: hal-01062739 https://hal.science/hal-01062739}

Submitted on 17 Sep 2014

HAL is a multi-disciplinary open access archive for the deposit and dissemination of scientific research documents, whether they are published or not. The documents may come from teaching and research institutions in France or abroad, or from public or private research centers.
L'archive ouverte pluridisciplinaire HAL, est destinée au dépôt et à la diffusion de documents scientifiques de niveau recherche, publiés ou non, émanant des établissements d'enseignement et de recherche français ou étrangers, des laboratoires publics ou privés. 


\title{
Continuous-discrete time observers for a class of MIMO nonlinear systems
}

\author{
M. Farza ${ }^{1}$, M. M'Saad ${ }^{1}$, M.L. Fall ${ }^{1}$, E. Pigeon ${ }^{1}$, O. Gehan ${ }^{1}$ and R. Mosrati ${ }^{2}$
}

\begin{abstract}
In this paper, we investigate the possibility of designing an observer for a class of continuous-time dynamical systems with non-uniformly sampled measurements. More specifically, we propose an observer with a time varying gain witch converges exponentially under some conditions on the sampling partition diameter. The proposed observer is an impulsive system since it is described by a set of differential equations with instantaneous state impulses corresponding to the measured samples and their estimates. As it is customarily done in the literature, we show that such an impulsive system can be split into two subsystems and be put under the form of a hybrid system which is designed using a continuoustime observer together with an inter-sample output predictor. Simulations results involving a typical bioreactor are given to show the effectiveness of the proposed observer.
\end{abstract}

Key words: Nonlinear observers, sampled-data observers, continuous-discrete time observers, high gain observers.

\section{INTRODUCTION}

The observer design problem for nonlinear dynamical systems has been paid a lot of attention over the last decades. It is however worth mentioning that most of the available results on nonlinear observers design deal with continuoustime measurements ([1], [2], [3], [4], [5]). Furthermore, the digital implementation of these continuous-time observers is generally carried out without any redesign using relatively small sampling periods as the stability and the convergence properties of the sampled continuous-time observer may be lost in the case of relatively large sampling periods. This is, the search for alternative design methodologies might be necessary to deal with the long standing digital implementation issue. Among these approaches, one can cite those whereby the observer design is based on exact or approximate discrete-time descriptions of the systems dynamics ([6], [7], [8]). This approach does not allow to take into consideration the inter-sampled dynamics, which is completely lost as pointed out in [9]. Moreover, the underlying discrete-time representation of the system may suffer from possible errors that might occur during the sampling scheduling process. Nevertheless, such an issue can be handled by appropriately redesigning the continuous-time observer and by considering the fact that the outputs measurements are available only at sampling instants. An earlier

Research supported by the European Commission under the project HYCON2 Highly-complex and networked control systems. ${ }^{1}$ The authors are with the GREYC Laboratory, UMR 6072 CNRS, Université de Caen and ENSICAEN, 6 Bd Maréchal Juin, 14050 Caen Cedex, France. e-mail: mondher.farza@ unicaen.fr. ${ }^{2}$ R. Mosrati is with ERPCB (Equipe de recherche en Physico-Chimie et Biotechnologie) of Caen. email. Ridha.mosrati@unicaen.fr contribution, in this context, has been made using a high gain observer for a class of nonlinear systems that are observable for any input [10]. The design has been firstly carried out by assuming continuous-time output measurements before being appropriately modified to handle the case where these measurements are only available at sampling instants. Based on the aforementioned contribution, many other observers have been proposed for specific classes of continuous-time systems with discrete-time outputs measurements ([11], [12], [13]).

A more promising approach to cope with the non availability of the output measurements between the sampling instants has been proposed in [9]. It consisted in employing a continuous time-observer using a suitable output predictor. The involved output prediction is obtained as the solution of a scalar ordinary differential equation (ODE) between two successive sampling instants with the value of the measured output sample as initial condition. The underlying observer is a hybrid system that inherits the properties of the continuous time observer for relatively fast sampling rates.

Another observer design approach for a particular class of nonlinear systems with sampled measurements has been proposed in [14]. It consists in using an impulsive observer where the output error correction term is expressed as the product of a constant gain by the difference between the estimated and measured values of the last output sample, respectively. The observer convergence analysis as well as the determination of its gain are obtained using LMI tools similar to those described in [15].

In this paper, we propose a continuous-discrete time observer for a class of multi-input and multi-output (MIMO) nonlinear systems, with sampled measurements, that are observable for any input. The proposed observer is obtained from a redesigned version of a purely continuous-time one that assumes continuous-time measurements. As for the impulsive observer given in [14], the corrective term of the proposed observer is a function of the difference between the estimated and actual output values at the last sampling instant. However, unlike in [14], the multiplicative observer gain is time-varying and depends on the sampling periods. In particular, it is shown that the proposed impulsive observer can be written under the form of a hybrid system as the continuous-discrete time observer proposed in [9]. Indeed, the equations of the impulsive observer can be split into two subsystems: the first subsystem has the same structure as the purely continuous time observer where the non available outputs are replaced by appropriate predictions provided by the second subsystem. 
The paper is organized as follows. In the next section, the class of systems that shall be considered throughout this paper is introduced with some notations together and a brief recall on the design of a continuous-time high gain observer for the considered class of systems. In section 3, the design of the impulsive continuous-discrete time observer is firstly detailed with full convergence analysis. Then, it is shown that the proposed impulsive observer can be written under the form of a hybrid system, allowing thereby to emphasize the relationship between this observer and the continuous high gain observer. Simulations results are given in section 4 to highlight the performances of the proposed observers. Finally, some concluding remarks are given in section 5.

\section{PROBLEM FORMULATION AND PRELIMINARIES}

Consider MIMO systems that are diffeomorphic to the following form:

$$
\left\{\begin{array}{l}
\dot{x}(t)=A x(t)+\varphi(u(t), x(t)) \\
y_{k}=C x\left(t_{k}\right)=x^{1}\left(t_{k}\right), \forall k \in \mathbb{N}
\end{array}\right.
$$

with

$$
\begin{aligned}
& x=\left(\begin{array}{c}
x^{1} \\
x^{2} \\
\vdots \\
x^{q}
\end{array}\right) ; \varphi(u, x)=\left(\begin{array}{c}
\varphi^{1}\left(u, x^{1}\right) \\
\varphi^{2}\left(u, x^{1}, x^{2}\right) \\
\vdots \\
\varphi^{q-1}\left(u, x^{1}, \ldots, x^{q-1}\right) \\
\varphi^{q}(u, x)
\end{array}\right) ; \\
& A=\left[\begin{array}{ccccc}
0_{p} & I_{p} & 0_{p} & & 0_{p} \\
\vdots & \ddots & I_{p} & & \\
0_{p} & & \ddots & \ddots & 0_{p} \\
0_{p} & & & \ddots & I_{p} \\
0_{p} & \cdots & & 0_{p} & 0_{p}
\end{array}\right] \\
& C=\left[I_{p}, 0_{p}, \ldots, 0_{p}\right]
\end{aligned}
$$

where the state $x \in \mathbb{R}^{n}$ with $x^{j} \in \mathbb{R}^{p}, j=1, \ldots, q$; the input $u(t) \in U$, a compact subset of $\mathbb{R}^{s}$ and the output $y \in \mathbb{R}^{p}$ is available at time instants $t_{k}$ satisfying

$$
\begin{aligned}
& 0 \leq t_{0}<\ldots<t_{k}<t_{k+1}<\ldots \text { with } \lim _{k \rightarrow \infty} t_{k}=\infty \\
& \text { and time-varying intervals } \tau_{k}=t_{k+1}-t_{k}
\end{aligned}
$$

System (1) may seem as being very particular since it assumes a non prime dimension $(n=p q)$ and in all the $q$ blocs, the sub-blocks $x^{j}$ have the same dimension $p$. In fact, it has been shown in [16] that system (1) is a normal form that characterizes a class of nonlinear systems that are observable for any input and that can be put through an injective map under the form (1) (see e.g. [16], [17] for more details).

Our objective consists in designing an exponential continuous-discrete time observer that provides continuoustime estimates of the whole state of system (1) from the outputs measurements that are available at sampling instants, only. One also aims at providing an expression of the upper bound of the sampling partition diameter under which the proposed observer converges exponentially. These objectives shall be achieved in the subsequent sections.

The observer design requires some assumptions that shall be stated in due courses. At this step, one assumes the following: (A1) The function $\varphi^{i}(u, x)$ are globally Lipschitz with respect to $x$ uniformly in $u$ i.e.

$$
\forall x, \bar{x} \in \mathbb{R}^{n}:\left\|\varphi^{i}(u, x)-\varphi^{i}(u, \bar{x})\right\| \leq L\|x-\bar{x}\|
$$

where $L>0$ is the Lipschitz constant.

Before proposing the continuous-discrete time impulsive observer, one shall recall the main steps of a continuous time high gain observer design for system (1). The main outlines of the underlying convergence analysis shall also be sketched. This allows to show later that the continuousdiscrete time impulsive observer can be interpreted as a redesigned version of the introduced continuous-time one. More specifically, one will consider the continuous-time observer that has been proposed for system (1) in ([18], [19]). The latter is given by

$\dot{\hat{x}}(t)=A \hat{x}(t)+\varphi(u(t), \hat{x}(t))-\theta \Delta_{\theta}^{-1} K(C \hat{x}(t)-y(t))$

where $\hat{x}=\left(\begin{array}{c}\hat{x}^{1} \\ \hat{x}^{2} \\ \vdots \\ \hat{x}^{q}\end{array}\right) ; K$ is a gain matrix and is chosen such that

$$
\bar{A} \triangleq A-K C
$$

is Hurwitz and $\Delta_{\theta}$ is the following diagonal matrix:

$$
\Delta_{\theta}=\operatorname{diag}\left(I_{p}, \frac{1}{\theta} I_{p}, \ldots, \frac{1}{\theta^{q-1}} I_{p}\right)
$$

where $\theta \geq 1$ is a real design parameter.

A detailed proof of the exponential convergence of the observation error can be found in [19]. For clarity purposes and since the link between the design of this observer and that of the continuous-discrete time one shall be raised, one proposes to briefly recall the main outlines of the proof. Indeed, let $\tilde{x}=\hat{x}-x$ be the observation error. One has

$$
\dot{\tilde{x}}=\left(A-\theta \Delta_{\theta}^{-1} K C\right) \tilde{x}+\varphi(u, \hat{x})-\varphi(u, x)
$$

Let $\bar{x}=\Delta_{\theta} \tilde{x}$. Taking into account the following identities

$$
\Delta_{\theta} A \Delta_{\theta}^{-1}=\theta A \text { and } C \Delta_{\theta}^{-1}=C
$$

one gets

$$
\dot{\bar{x}}=\theta \bar{A} \bar{x}+\Delta_{\theta}(\varphi(u, \hat{x})-\varphi(u, x))
$$

Now, since $\bar{A}$ is Hurwitz, there exist a symmetric positive definite matrix $P$ and a positive real $\mu$ such that

$$
P \bar{A}+\bar{A}^{T} P \leq-2 \mu I_{n}
$$

Let us show that $V(\bar{x})=\bar{x}^{T} P \bar{x}$ is a Lyapunov function for system (12). Indeed, one has

$$
\begin{aligned}
\dot{V}(\bar{x}) & =2 \theta \bar{x}^{T} P \bar{A} \bar{x}+2 \bar{x}^{T} P \Delta_{\theta}(\varphi(u, \hat{x})-\varphi(u, x)) \\
& \leq-2 \mu \theta\|\bar{x}\|^{2}+2 \bar{x}^{T} P \Delta_{\theta}(\varphi(u, \hat{x})-\varphi(u, x))
\end{aligned}
$$


According to the Lipschitz assumption and the triangular structure of $\varphi$, one can show that ([19], [16]):

$$
2 \bar{x}^{T} P \Delta_{\theta}(\varphi(u, \hat{x})-\varphi(u, x)) \leq 2 L \sqrt{n} \lambda_{M}\|\bar{x}\|^{2}
$$

where $L$ is the Lipschitz constant of $\varphi$ and $\lambda_{M}$ (resp. $\lambda_{m}$ ) is the largest (resp. smallest) eigenvalue of $P$.

Combining (14) and (15), one gets

$$
\dot{V}(\bar{x}) \leq-\left(2 \mu \theta-2 L \sqrt{n} \lambda_{M}\right)\|\bar{x}\|^{2}
$$

Now, by choosing $\theta$ such that $2 \mu \theta-2 L \sqrt{n} \lambda_{M}>\mu \theta$ i.e.

$$
\theta>\theta_{0} \triangleq \frac{2 L \sqrt{n} \lambda_{M}}{\mu}
$$

one gets

$$
V(\bar{x}(t)) \leq e^{-\frac{\mu \theta}{\lambda_{M}}\left(t-t_{0}\right)} V\left(\bar{x}\left(t_{0}\right)\right)
$$

This ends the proof.

\section{SyNTHESIS OF THE CONTINUOUS-DISCRETE TIME OBSERVER}

The candidate impulsive continuous-discrete time observer takes the following form

$$
\begin{aligned}
\dot{\hat{x}}(t)= & A \hat{x}(t)+\varphi(u(t), \hat{x}(t)) \\
& -\Delta_{\theta}^{-1} K e^{-\theta K^{1}\left(t-t_{k}\right)}\left(C \hat{x}\left(t_{k}\right)-y\left(t_{k}\right)\right), \quad k \in \mathbb{N}(18)
\end{aligned}
$$

where $\hat{x}=\left(\begin{array}{c}\hat{x}^{1} \\ \hat{x}^{2} \\ \vdots \\ \hat{x}^{q}\end{array}\right)$; the matrix $K=\left(\begin{array}{c}K^{1} \\ K^{2} \\ \vdots \\ K^{q}\end{array}\right)$, where the $K^{i}$ s are $p \times p$ square matrices, is such that the matrix $\bar{A}$ defined as in (8) is Hurwitz, the matrix $\Delta_{\theta}$ is defined in (9) and $\theta \geq 1$ is a design parameter.

Under Assumption (A1), our main result can be stated as follows:

Theorem 1. For every bounded input, there exists $\theta_{0}>0$; for every $\theta>\theta_{0}$; there exists $\tau_{M}>0$ and $\chi(\theta)>0$ with $\tau_{M}<\frac{1}{\chi(\theta)}$ such that for all $k \in \mathbb{N}$ with $t_{k+1}-t_{k} \leq \tau_{m}$, the observation error $\hat{x}(t)-x(t)$ where $x$ and $\hat{x}$ are the respective trajectories of systems (1) and (18) exponentially converges to zero.

Notice that the parameter $\tau_{M}$ is the upper diameter of the sampling partition (i.e. the maximum possible value of the sampling period) under which the exponential convergence of the observation error is guaranteed.

\section{A. Proof of Theorem 1.}

Let $\tilde{x}(t)=\hat{x}(t)-x(t)$ be the observation error where $t \in$ $\left[t_{k} t_{k+1}[\right.$. One has

$$
\dot{\tilde{x}}(t)=A \tilde{x}+\varphi(u, \hat{x})-\varphi(u, x)-\theta \Delta_{\theta}^{-1} K e^{-K^{1} \theta\left(t-t_{k}\right)} C \tilde{x}\left(t_{k}\right)
$$

Consider the following change of variables $\bar{x}=\Delta_{\theta} \tilde{x}$. Using the identities (11), on gets

$\dot{\bar{x}}(t)=\theta A \bar{x}+\Delta_{\theta}(\varphi(u, \hat{x})-\varphi(u, x))-\theta K e^{-K^{1} \theta\left(t-t_{k}\right)} C \bar{x}\left(t_{k}\right)$
Let us consider the following candidate quadratic Lyapunov function

$$
V(\bar{x})=\bar{x}^{T} P \bar{x}
$$

where $P$ is defined as in (13).

Proceeding as in the continuous case, one can show that

$$
\begin{aligned}
\dot{V}(\bar{x}) \leq & -2 \theta \mu\|\bar{x}\|^{2}+2 \theta \bar{x}^{T} P K C \bar{x}+2 L \sqrt{n} V_{1}(\bar{x}) \\
& -2 \theta \bar{x}^{T} P K e^{-K^{1} \theta\left(t-t_{k}\right)} C \bar{x}\left(t_{k}\right) \\
\leq & -\left(2 \theta \mu-2 L \sqrt{n} \lambda_{M}\right)\|\bar{x}\|^{2}+2 \theta \bar{x}^{T} P K z
\end{aligned}
$$

where

$$
\begin{aligned}
z(t) & \triangleq C \bar{x}-e^{-K^{1} \theta\left(t-t_{k}\right)} C \bar{x}\left(t_{k}\right) \\
& =\bar{x}^{1}(t)-e^{-K^{1} \theta\left(t-t_{k}\right)} \bar{x}^{1}\left(t_{k}\right)
\end{aligned}
$$

Notice that, one has $z\left(t_{k}\right)=0$. Moreover, the time derivative of $z$ can be expressed as follows

$$
\begin{aligned}
\dot{z}(t) & =\dot{\bar{x}}^{1}(t)+K^{1} \theta e^{-K^{1} \theta\left(t-t_{k}\right)} \bar{x}^{1}\left(t_{k}\right) \\
& =\theta \bar{x}^{2}+\varphi^{1}\left(u, \hat{x}^{1}\right)-\varphi^{1}\left(u, x^{1}\right)
\end{aligned}
$$

Integrating (22) from $t_{k}$ to $t$ and from the fact that $z\left(t_{k}\right)=0$, one gets:

$$
z(t)=\int_{t_{k}}^{t}\left(\theta \bar{x}^{2}(s)+\varphi^{1}\left(u, \hat{x}^{1}(s)\right)-\varphi^{1}\left(u, x^{1}(s)\right)\right) d s
$$

and hence

$$
\begin{aligned}
\|z(t)\| & \leq \int_{t_{k}}^{t}\left(\theta\left\|\bar{x}^{2}(s)\right\|+L\left\|\bar{x}^{1}(s)\right\|\right) d s \\
& \leq(\theta+L) \int_{t_{k}}^{t}\|\bar{x}(s)\| d s
\end{aligned}
$$

By choosing $\theta$ as in the continuous case, i.e. satisfying (17), inequality (20) becomes

$$
\begin{aligned}
\dot{V}(\bar{x}(t)) \leq & -\mu \theta\|\bar{x}\|^{2}+2 \theta \bar{x}^{T}(t) P K z(t) \\
\leq & -\frac{\mu \theta}{\lambda_{M}} V(\bar{x}(t))+2 \theta \sqrt{\lambda_{M}} \sqrt{V(\bar{x}(t))}\|K\|\|z(t)\| \\
\leq & -\frac{\mu \theta}{\lambda_{M}} V(\bar{x}(t))+2 \theta \sqrt{\frac{\lambda_{M}}{\lambda_{m}}}\|K\|(L+\theta) . \\
& \sqrt{V(\bar{x}(t))} \int_{t_{k}}^{t} \sqrt{V(\bar{x}(s))} d s
\end{aligned}
$$

or equivalently

$$
\begin{aligned}
\frac{\dot{V}(\bar{x}(t))}{2 \sqrt{V(\bar{x}(t))} \leq}- & -\frac{\mu \theta}{2 \lambda_{M}} \sqrt{V(\bar{x}(t))}+\theta \sqrt{\frac{\lambda_{M}}{\lambda_{m}}}\|K\| . \\
& (L+\theta) \int_{t_{k}}^{t} \sqrt{V(\bar{x}(s))} d s
\end{aligned}
$$

Integrating the above inequation from $t_{k}$ to $t$, one gets:

$$
\begin{aligned}
\sqrt{V(\bar{x}(t))} \leq & e^{-\frac{\mu \theta}{2 \lambda_{M}}\left(t-t_{k}\right)} \sqrt{V\left(\bar{x}\left(t_{k}\right)\right)} \\
& +\theta \sqrt{\frac{\lambda_{M}}{\lambda_{m}}}\|K\|(L+\theta) \int_{t_{k}}^{t} e^{-\frac{\mu \theta}{2 \lambda_{M}}(t-s)} \\
& \left(\int_{t_{k}}^{s} \sqrt{V(\bar{x}(\xi))} d \xi\right) d s
\end{aligned}
$$


Now, one has

$$
\begin{aligned}
& \int_{t_{k}}^{t} e^{-\frac{\mu \theta}{2 \lambda_{M}}(t-s)} \int_{t_{k}}^{s} \sqrt{V(\bar{x}(\xi))} d \xi d s \\
& \leq \int_{t_{k}}^{t} \sqrt{V(\bar{x}(\xi))} d \xi \int_{t_{k}}^{t} e^{-\frac{\mu \theta}{2 \lambda_{M}}(t-s)} d s \\
& \leq e^{-\frac{\mu \theta}{2 \lambda_{M}}\left(t-t_{k}\right)} \frac{\left(e^{\frac{\mu \theta}{2 \lambda_{M}} \tau_{M}}-1\right)}{\frac{\mu \theta}{2 \lambda_{M}}} \int_{t_{k}}^{t} \sqrt{V(\bar{x}(\xi))} d \xi
\end{aligned}
$$

Using the bound given by (28) in (26), one gets

$$
\begin{aligned}
\sqrt{V(\bar{x}(t))} \leq & e^{-\frac{\mu \theta}{2 \lambda_{M}}\left(t-t_{k}\right)} \sqrt{V\left(\bar{x}\left(t_{k}\right)\right)} \\
& +c e^{-\frac{\mu \theta}{2 \lambda_{M}}\left(t-t_{k}\right)} \int_{t_{k}}^{t} \sqrt{V(\bar{x}(\xi))} d \xi
\end{aligned}
$$

where

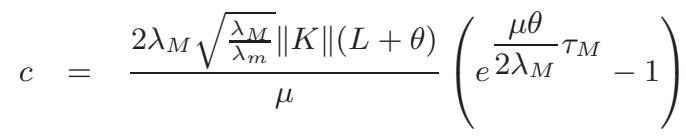

Multiplying each side of inequality (29) by $e^{\frac{\mu \theta}{2 \lambda_{M}}\left(t-t_{k}\right)}$ leads to

$$
\begin{aligned}
e^{\frac{\mu \theta}{2 \lambda_{M}}\left(t-t_{k}\right)} \sqrt{V(\bar{x}(t))} \leq & \sqrt{V\left(\bar{x}\left(t_{k}\right)\right)} \\
& +c \int_{t_{k}}^{t} \sqrt{V(\bar{x}(\xi))} d \xi
\end{aligned}
$$

By setting

$$
U(t)=e^{\frac{\mu \theta}{2 \lambda_{M}}\left(t-t_{k}\right)} \sqrt{V(\bar{x}(t))}
$$

inequation (31) becomes

$$
\begin{aligned}
U(t) & \leq \sqrt{V\left(\bar{x}\left(t_{k}\right)\right)}+c \int_{t_{k}}^{t} e^{-\frac{\mu \theta}{2 \lambda_{M}}\left(\xi-t_{k}\right)} U(\xi) d \xi \\
& \leq \sqrt{V\left(\bar{x}\left(t_{k}\right)\right)}+c \int_{t_{k}}^{t} U(\xi) d \xi
\end{aligned}
$$

Using Gronwall's lemma, one gets

$$
U(t) \leq e^{c\left(t-t_{k}\right)} \sqrt{V\left(\bar{x}\left(t_{k}\right)\right)}
$$

and one comes back to $\sqrt{V}$ using (32) to obtain

$$
\sqrt{V(\bar{x}(t))} \leq e^{-\left(\frac{\mu \theta}{2 \lambda_{M}}-c\right)\left(t-t_{k}\right)} \sqrt{V\left(\bar{x}\left(t_{k}\right)\right)}
$$

Since $\bar{x}$ and $V(\bar{x})$ are continuous with respect to their arguments, one has $\sqrt{V\left(\bar{x}\left(t_{k}\right)\right)}=\sqrt{V\left(\bar{x}\left(t_{k}-\right)\right)}$ and one can easily deduce from (35) the following

$$
\sqrt{V(\bar{x}(t))} \leq e^{-\left(\frac{\mu \theta}{2 \lambda_{M}}-c\right)\left(t-t_{0}\right)} \sqrt{V\left(\bar{x}\left(t_{0}\right)\right)}
$$

To end the proof, one has to exhibit the condition under which the term $\left(\frac{\mu \theta}{2 \lambda_{M}}-c\right)$, is positive. Using the expression of the constant $c$ given by (30), such a condition shall be satisfied if:

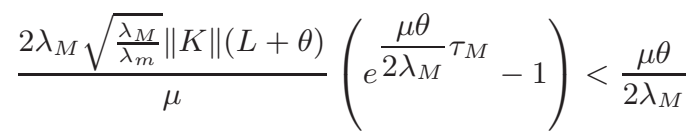

or equivalently

$$
\tau_{M}<\frac{2 \lambda_{M}}{\mu \theta} \operatorname{Ln}\left(1+\frac{\left(\frac{\mu}{2 \lambda_{M}}\right)^{2} \theta}{\sqrt{\frac{\lambda_{M}}{\lambda_{m}}}\|K\|(L+\theta)}\right)
$$

This ends the proof.

It is important to note that condition (38) may be very restrictive and it only provides an upper bound for the sampling partition diameter. Nevertheless, many simulation experiments have shown that the proposed continuous-discrete time observer perform well for sampling periods that are higher than the theoretical upper bound given by (38).

Remark: If a system $\dot{x}(t)=f(u(t), x(t)), y\left(t_{k}\right)=h\left(x\left(t_{k}\right)\right)$ is put under form (1) through a diffeomorphism $z=\Phi(x)$, then the equation of the continous-discrete time observer (18) can be written in the original coordinates in ' $x$ ' as follows:

$$
\begin{aligned}
\dot{\hat{x}}(t)= & f(u(t), \hat{x}(t))-\theta e^{-\theta K^{1}\left(t-t_{k}\right)} \\
& \left(\frac{\partial \Phi}{\partial x}(\hat{x}(t))\right)^{-1} \Delta_{\theta}^{-1} K\left(h\left(\hat{x}\left(t_{k}\right)\right)-y\left(t_{k}\right)\right)
\end{aligned}
$$

\section{B. The output prediction form of the observer}

Equation (18) puts forward the impulsive form of the proposed observer. We shall show that this observer can be split into two subsystems: the structure of the first subsystem is similar to that of the continuous-time observer (7) where the unavailable output is replaced by an appropriate prediction provided by the second subsystem. Indeed, consider the following continuous-time dynamical system:

$$
\dot{\hat{x}}(t)=A \hat{x}(t)+\varphi(u(t), \hat{x}(t))-\theta \Delta_{\theta}^{-1} K(C \hat{x}(t)-\omega(t))
$$

where $\hat{x}, K$ are as in (18) and $\theta \geq 1$ is a design parameter. The structure of this system is very similar to that of the continuous time observer (7) with the difference that the term corresponding to the observation error in (7), $(C \hat{x}(t)-y(t))$, is replaced in (40) by the term $(C \hat{x}(t)-\omega(t))$ where $\omega(t)$ is a new variable and it corresponds to the prediction of the outputs. Its dynamics is described by the following Ordinary Differential Equation (ODE):

$$
\left\{\begin{array}{rll}
\text { For } t_{k} \leq \quad & t \quad<t_{k+1}: \\
\dot{\omega}(t) & =\hat{x}^{2}(t)+\varphi^{1}\left(u(t), \hat{x}^{1}(t)\right) \\
\text { with } \omega\left(t_{k}\right) & =C x\left(t_{k}\right)=y\left(t_{k}\right)
\end{array}\right.
$$

The dynamics of the outputs prediction $\omega$ is very similar to that of the outputs estimate $\hat{x}^{1}(t)$ that can be written (according to system (40)) as follows:

$\dot{\hat{x}}^{1}(t)=\hat{x}^{2}(t)+\varphi^{1}\left(u(t), \hat{x}^{1}(t)\right)-\theta K^{1}(C \hat{x}(t)-\omega(t))$

Recall that contrarily to the ODE (41) that has to be reinitialized at each sampling instant $t_{k}$ and then resolved for 
$t \in\left[t_{k}, t_{k+1}[\right.$, equation (42) or more generally system (40) is initialized only once at $t_{0}$ and then resolved for all $t \geq t_{0}$. Set

$$
\xi(t)=C \hat{x}(t)-\omega(t)=\hat{x}^{1}(t)-\omega(t)
$$

and let us focus on the dynamics of $\xi$. Indeed, according to (41) and (42), one has:

$$
\dot{\xi}(t)=-\theta K^{1} \xi(t)
$$

Integrating (44) from $t_{k}$ to $t<t_{k+1}$ yields

$$
\xi(t)=e^{-\theta K^{1}\left(t-t_{k}\right)} \xi\left(t_{k}\right)
$$

Now, from the fact that $\omega\left(t_{k}\right)=x^{1}\left(t_{k}\right)$, one has

$$
\begin{aligned}
\xi\left(t_{k}\right) & =\hat{x}^{1}\left(t_{k}\right)-\omega\left(t_{k}\right) \\
& =\hat{x}^{1}\left(t_{k}\right)-x^{1}\left(t_{k}\right)=\hat{x}^{1}\left(t_{k}\right)-y\left(t_{k}\right)
\end{aligned}
$$

Combining (45) and (46) gives

$$
\xi(t)=e^{-\theta K^{1}\left(t-t_{k}\right)} C \tilde{x}\left(t_{k}\right)
$$

Finally, substituting $C \hat{x}(t)-\omega(t) \triangleq \xi(t)$ in equation (40) by its expression given by (47), one gets:

$$
\begin{aligned}
\dot{\hat{x}}(t)= & A \hat{x}(t)+\varphi(u(t), \hat{x}(t)) \\
& -\theta \Delta_{\theta}^{-1} e^{-\theta K^{1}\left(t-t_{k}\right)} K\left(C \hat{x}\left(t_{k}\right)-y\left(t_{k}\right)\right)
\end{aligned}
$$

and we meet observer (18).

\section{EXAMPLE}

The performance of the proposed observer shall be illustrated through an observer design involving a typical bioreactor. We consider a simple microbial culture which involves a single biomass $x_{1}$ growing on a single substrate $x_{2}$. The bioprocess is supposed to be continuous with a dilution rate $u(t)$ and an input substrate concentration $s_{i n}(t)$. The specific growth rate is assumed to follow the Contois model ([20]). The mathematical dynamical model of the process is constituted by the following two mass balance equations associated to $x_{1}$ and $x_{2}$, respectively:

$$
\left\{\begin{aligned}
\dot{x}_{1}(t) & =\frac{\mu^{\star} x_{1}(t) x_{2}(t)}{K_{c} x_{1}(t)+x_{2}(t)}-u(t) x_{1}(t) \\
\dot{x}_{2}(t) & =\frac{-k \mu^{\star} x_{1}(t) x_{2}(t)}{K_{c} x_{1}(t)+x_{2}(t)}+u(t)\left(s_{i n}(t)-x_{2}(t)\right) \\
y\left(t_{k}\right) & =x_{1}\left(t_{k}\right)
\end{aligned}\right.
$$

where $x_{1}$ and $x_{2}$ respectively denote the concentration of the biomass and the substrate, $\mu^{\star}$ and $K_{c}$ are the Contois law parameters while $k$ is a yield coefficient. The measurements of the biomass concentration are supposed to be available with at time interval $\tau$ and the objective is to estimate the actual biomass concentration together with that of the substrate from the available discrete measurements.

System (49) has been considered in [21] where the authors exhibited a compact set $X \in \mathbb{R}^{2}$ which is positively invariant under the dynamics of (49). Moreover, it was shown that the following function $\Phi: X \longrightarrow \Phi(X)$, $x=\left(\begin{array}{c}x_{1} \\ x_{2}\end{array}\right) \mapsto z=\Phi(x)=\left(\begin{array}{ccc}z_{1} & = & x_{1} \\ z_{2} & = & \frac{\mu^{\star} x_{1} x_{2}}{K_{C} x_{1}+x_{2}}\end{array}\right)$ is a diffeomorphism from $X$ onto its image. System (49) can be written in the new coordinates $z$ under form (1). As a result, an observer proposed in this paper can be used to achieve the continuous state estimation of the state from the available discrete measurements. The equations of the observer in the original equations can be written under form (39)

Simulations have been carried out by assuming a uniform sampling partition i.e. $t_{k}=k \tau$. The following values are used in simulation:

$$
\begin{gathered}
\mu^{\star}=1\left(\min ^{-1}\right), K_{C}=1, k=1, u=0.08\left(\min ^{-1}\right) \\
s_{i n}=0.1\left(k g m^{-3}\right)
\end{gathered}
$$

Notice that the equilibrium point is $\left(x_{1,0}, x_{2,0}\right)=$ $(0.092,0.008)$. The simulation of the observer has been carried out using pseudo data measurements issued from the simulation of system (49) with the point equilibrium as an initial condition. The initial condition of the observer is such that the one corresponding to the substrate concentration is perturbed by $50 \%$ from the steady state value. Many simulations have been carried out and they show that satisfactory estimates are obtained as long as $\tau \leq 5.5$. Simulation results are obtained by setting $\tau=2$. The values of the design parameters are $\theta=2$ and $\rho=20$, respectively. Notice that the choice of $\tau$ and $\theta$ should meet condition of Theorem 1 which is may be restrictive. However in practice values $\tau$ bigger than that allowed by Theorem 1 may be used and satisfactory estimates will be obtained. The gain vector is chosen equal to $K=\left(\begin{array}{l}2 \\ 1\end{array}\right)$ so that both poles of the matrix $\bar{A}$ are located in -1 . The available samples corresponding to the output $x_{1}$ are given in figure 1 and they are compared to the continuous estimate of this output provided by the observer. Similarly, the unknown trajectory of $x_{2}$ is compared to its estimate in figure 2. Notice that the state estimates quickly converge to the unknown states confirming thereby the theoretical results.

\section{CONCLUSion}

A continuous-discrete time observer has been proposed for a class of systems that are observable for any input. The structure of this observer is simple since it consists in a copy of the model with a corrective term that is updated at every sampling instant. The exponential convergence to zero of the observation error has been established under certain conditions on the upper bound of the sampling partition diameter. This observer has been firstly presented under an impulsive form before being put under the form of a hybrid system synthesized using a continuous-time design with an inter sample output predictor.

The proposed design can be extended to a more general class of observable systems including system (1). It can 


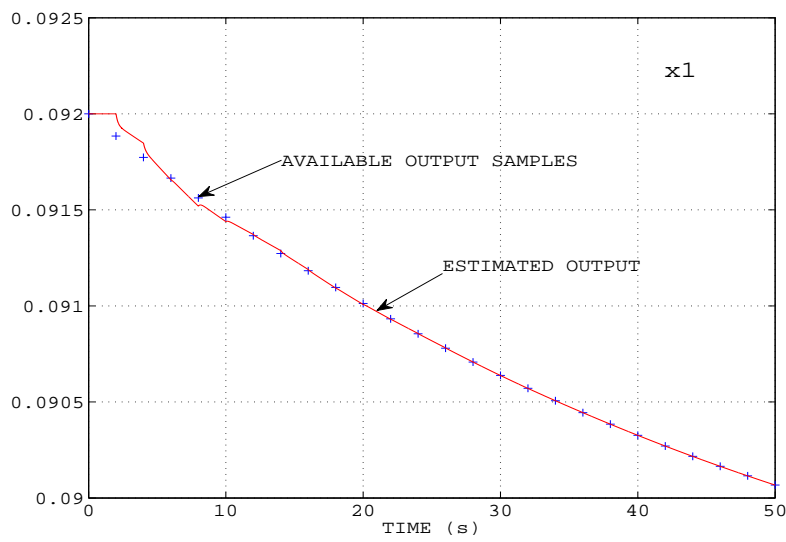

Fig. 1. Continuous estimates of the outputs from they measured samples.

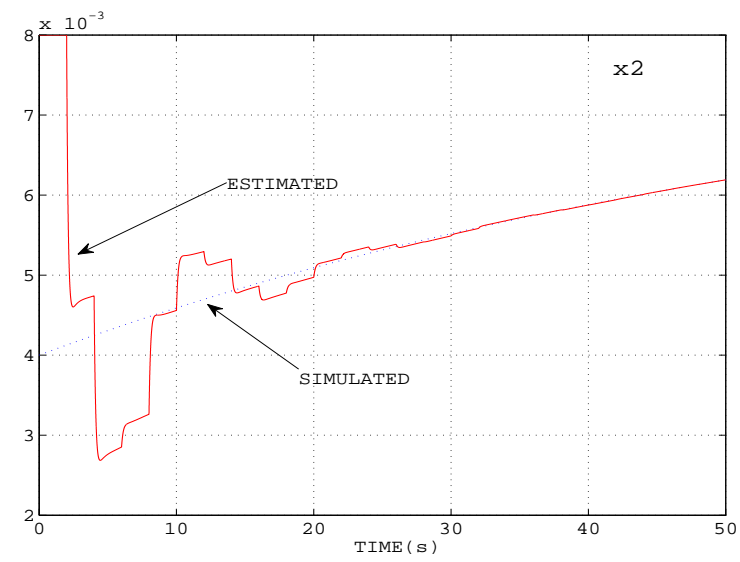

Fig. 2. Continuous estimates of the non measured states.

also be exploited to derive continuous-discrete time adaptive observers to jointly estimate the state and some unknown parameters from the adaptive observer proposed in [17]. Another problem that has not be considered in this paper deals with the case where sampling is accompanied with long output delays. Such a problem has been addressed in the context of stabilization (see e.g. [22] and references therein) but to the authors' best knowledge, there is no significant available results for this problem in terms of observation and the (few) available ones rather deal with continuoustime measurements [23], [24], [25], [26]. All these issues are challenging and shall be investigated in further works.

\section{REFERENCES}

[1] J. Gauthier and I. Kupka, Deterministic Observation Theory and Applications. Cambridge University Press, 2001.

[2] N. Kazantzis and C. Kravaris, "Nonlinear observer design using lyapunov's auxiliary theorem," Systems \& Control Letters, vol. 34, pp. 241-247, 1998.

[3] M. Farza, M. M'Saad, M. Triki, and T. Maatoug, "High gain observer for a class of non-triangular systems," Systems \& Control Letters, vol. 60, no. 1, pp. 27-35, 2011.

[4] R. Rajamani, "Observers for Lipschitz Nonlinear Systems," IEEE Transactions on Automatic Control, vol. 43, no. 3, pp. 397-401, 1998.
[5] A. J. Krener and A. Isidori, "Linearization by output injection and nonlinear observers," Syst. Contr. Lett., vol. 3, pp. 47-52, 1983.

[6] M. Arcak and D. Nesic, "A framework for nonlinear sampled-data observer design via approximate discrete-time models and emulation," Automatica, vol. 40, pp. 1931-1938, 2004.

[7] M. Farza, K. Busawon, and H. Hammouri, "Simple Nonlinear Observers for On-line Estimation of Kinetic Rates in Bioreactors," Automatica, vol. 34, no. 3, pp. 301-318, 1998.

[8] N. Krener and C. Kravaris, "Discrete-time nonlinear observer design using functional equations," Systems \& Control Letters, vol. 42, pp. 81-94, 2001.

[9] I. Karafyllis and C. Kravaris, "From continuous-time design to sampled-data design of observers," IEEE Transactions on Automatic Control, vol. 54, no. 9, pp. 2169-2174, 2009.

[10] F. Deza, E. Busvelle, J. Gauthier, and D. Rakotopara, "High gain estimation for nonlinear systems," Systems \& Control Letters, vol. 18 , pp. 295-299, 1992.

[11] M. Nadri, H. Hammouri, and C. A. Zaragoza, "Observer design for continuous-discrete time state affine systems up to output injection," European Journal of Control, vol. 10, no. 3, pp. 252-263, 2004.

[12] H. Hammouri, M. Nadri, and R. Mota, "Constant gain observer for continuous-discrete time uniformly observable systems," in Proc. of the the 45th IEEE Conference on Decision and Control, San Diego, CA, USA, 2006.

[13] V. Andrieu and M. Nadri, "Observer design for lipschitz systems with discrete-time measurements," in In Proc. 49th IEEE Conference on Decision and Control, Atlanta, Georgia USA, 2010.

[14] T. Raff, M. Kögel, and F. Allgöwer, "Observer with sample-andhold updating for Lipschitz nonlinear systems with nonuniformly sampled measurements," in Proc. of the American Control Conference, Washington,USA, 2008.

[15] P. Naghshtabrizi, J. Hespanha, and A. Teel, "Exponential stability of impulsive systems with application to uncertain sampled-data systems," Systems \& Control Letters, vol. 57, pp. 378-385, 2008.

[16] H. Hammouri and M. Farza, "Nonlinear observers for locally uniformly observable systems," ESAIM J. on Control, Optimisation and Calculus of Variations, vol. 9, pp. 353-370, 2003.

[17] M. Farza, M. M'Saad, T. Maatoug, and M. Kamoun, "Adaptive observers for nonlinearly parameterized class of nonlinear systems," Automatica, vol. 45, no. 10, pp. 2292-2299, 2008.

[18] F. Deza, E. Busvelle, and J. Gauthier, "Exponentially converging observers for distillation columns and internal stability of the dynamic output feedback," Chemical Engineering Science, vol. 47, no. 15/16, 1992.

[19] M. Farza, M. M'Saad, and L. Rossignol, "Observer design for a class of MIMO nonlinear systems," Automatica, vol. 40, no. 1, pp. 135-143, 2004.

[20] J. Bailey and D. Ollis, Biochemical Engineering Fundamentals. McGraw-Hill, New York, 1986.

[21] J. Gauthier, H. Hammouri, and S. Othman, "A simple observer for nonlinear systems - application to bioreactors," IEEE Trans. Auto. Control, vol. 37, no. 6, pp. 875-880, 1992.

[22] I. Karafyllis and M. Krstic, "Nonlinear stabilization under sampled and delayed measurements, and with inputs subject to delay and zeroorder hold," private correspondance, 2012.

[23] A. Germani, C. Manes, and P. Pepe, "A new approach to state observation of nonlinear systems with delayed output," IEEE Transactions on Automatic Control, vol. 47, no. 1, pp. 96-101, 2002.

[24] N. Kazantzis and R. A. Wright, "Nonlinear observer design in the presence of delayed output measurements," Systems \& Control Letters, vol. 54, no. 9, pp. 877-886, 2005.

[25] F. Cacace, A. Germani, and C. Manes, "An observer for a class of nonlinear systems with time varying observation delay," Systems \& Control Letters, vol. 59, no. 5, pp. 305-312, 2010.

[26] M. Farza, M. M'Saad, O. Gehan, and E. Pigeon, "Predictors design for nonlinear systems - application to state observation with delayed output measurements," IEEE Transactions on Automatic Control. Submitted, 2012 